\title{
Histopathological Evaluation on the Effect of Induced Hypertension Chemotherapy Presurgically Performed in Patients with Advanced Carcinoma of the Stomach
}

\author{
Megumi Nakamura* $\ddagger$, Tohru Takahashi*, Haruhiko \\ Sato $\dagger$, Masahiko Hoshi $\dagger$, Akira Wakui $\dagger$ and \\ Ryunosuke Kanamaru $\dagger$ \\ *Department of Pathology and $\dagger$ Department of Clinical \\ Cancer Chemotherapy, the Research Institute for \\ Tuberculosis and Cancer, Tohoku University, Sendai 980, \\ and $\ddagger$ Department of Pediatric Surgery, Tohoku University \\ School of Medicine, Sendai 980
}

\begin{abstract}
Nakamura, M., Takahashi, T., Sato, H., Hoshi, M., Wakui, A. and Kanamaru, R. Histopathological Evaluation on the Effect of Induced Hypertension Chemotherapy Presurgically Performed in Patients with Advanced Carcinoma of the Stomach. Tohoku J. Exp. Med., 1992, 167 (1), 27-37_ Stomachs resected from 13 patients with an advanced gastric carcinoma were examined histopathologically to evaluate the effect of induced hypertension chemotherapy (IHC) using angiotensin II. Eight of the 13 patients, randomly chosen, were treated presurgically by IHC with a regimen of 5-fluorouracil, adriamycin and mitomycin $\mathrm{C}$; the same regimen was used in the remaining five patients but without inducing hypertension. Clinical evaluation of the effect gave rise to the confirmation, presurgically, of complete response in three and partial response(PR) in two patients in the IHC group, whereas in the non-IHC group, the highest rating was $\mathrm{PR}$, which was attained in only one patient. Also in the histopathological assessment based on a five-step grading, the IHC group earned in average a higher score than the non-IHC group, with a difference proved significant by Wilcoxon' s test. A histological rating of Grade 3, the highest effectiveness, was given to three patients in the IHC group, in one of whom the resected stomach disclosed no viable carcinoma cells but only fibrotic areas replacing carcinoma. There was also a correlation between the clinical and histological ratings as proved to be significant by Spearman's test. We conclude that in gastric carcinoma, the effect of chemotherapy is enhanced by angiotensin II-IHC. —— histopathology; induced hypertension chemotherapy ; angiotensin II ; gastric carcinoma
\end{abstract}

Induced hypertension chemotherapy (IHC) is a technique developed by

Received April 6, 1992; revision accepted for publication April 22, 1992.

Address for reprint: M. Nakamura, M.D., Department of Pathology, the Research Institute for Tuberculosis and Cancer, Tohoku University, 4 Seiryomachi, Aoba-ku, Sendai, Miyagi 980, Japan. 
Suzuki et al. $(1981,1984)$ in an effort to enhance the effect of cancer chemotherapy. It exploits the peculiar microcirculation of cancer tissue, where, due to lack of autoregulation which is present in ordinary tissues, blood flow is susceptible to fluctuation: The flow strongly increases when the systemic blood pressure is temporarily elevated by angiotensin II. This is a phenomenon partially attributable to the peculiar structure of newly developed "tumor vessels" that are mostly devoid of mural smooth muscles (Suzuki et al. 1987). Thus, if anticancer drugs are given under a temporary hypertension induced by angiotensin, one can expect an enhanced and selective delivery of drugs to cancer.

A phase III study was performed during the four years 1986 to 1989 for angiotensin II (human type) synthesized by Toa Eiyo Co. Ltd., Tokyo for clinical use in IHC. This gave us an opportunity to examine histopathologically the effect of IHC in patients with advanced gastric carcinoma. In some of them, presurgical chemotherapy had been performed under angiotensin-induced hypertension, while in the remaining patients, it was done in an ordinary way. The effect of cancer chemotherapy has rarely been subjected to systematized histopathological evaluation of resected materials, and here we report the comparative analysis of the resected stomachs between the two groups.

\section{Materials and Methods}

\section{Examined cases}

Studied were surgical materials obtained at gastrectomy of thirteen patients who had an advanced gastric carcinoma. The patients all received presurgical chemotherapy based on a regimen comprising 5-fluorouracil, adriamycin and mitomycin $\mathrm{C}$. In eight of the patients, drugs were given under temporary hypertension induced by a simultaneous infusion of angiotensin II : we called these the IHC group. In the remaining five, i.e., the non-IHC group, chemotherapy was performed exactly with the same protocol except for not introducing hypertension (Sato et al. 1991). The patients were allotted either to the IHC or non-IHC group according to a randomizing procedure. Clinical examinations prior to chemotherapy suggested in all patients the presence of carcinoma invasion into neighboring organs, metastasis to lymph nodes or distant organs, making them look not in a condition suitable for immediate operation. However, at the time chemotherapy was finished as designed, the state of patients appeared to have improved more or less, at least clinically. In the total amount of drugs given, there was no essential difference between the two groups. For the regimen and period of chemotherapy, also see the report of Sato et al. (1991). From all the patients, gastric mucosal biopsy was taken preoperatively, revealing one of the variants of adenocarcinoma.

\section{Methods of analyses}

An excised stomach was opened along the greater curve and fixed in $10 \%$ formaldehyde in a flatly extended state. After fixation was completed, specimens for histological examination were taken so that the portion of the gastric wall including the main tumor was sequentially cut into serial parallel strips, about $5 \mathrm{~mm}$ in breadth. Then the specimens were embedded in ordinary paraffin, sectioned at $3 \mu \mathrm{m}$ thickness and stained with HE, ElasticaGoldner and PAS. Although in Cases 8 and 12 not a set of whole specimens were available, what remained of them included the portion of maximal extension, allowing us to obtain a sufficient information about the spread of tumor. 
TABLE 1. A standard for histpathological assessment of the effect of chemotherapy for gastric carcinoma ("The General Rules for the Gastric Cancer Study" by Japanese Society for the Study of Gastric Cancer, 11th ed. 1985)

[Grade 0] No effect

[Grade 1] Mild to moderate effect

1a] Only mild effect, with less than $1 / 3$ of carcinoma undergoing degeneration or necrosis

1b] Moderate effect, with $1 / 3$ to $2 / 3$ of carcinoma undergoing degeneration, necrosis or liquefaction

[Grade 2] Pronounced effect, with more than $2 / 3$ of carcinoma undergoing degeneration, necrosis, liquefaction or loss

[Grade 3] Complete disappearance of carcinoma cells, leaving granulation tissues or scar areas

Microscopically, the effect of chemotherapy was evaluated according to "The General Rules for the Gastric Cancer Study" by Japanese Research Society for Gastric Cancer (11th ed. 1985). It advises pathologists to conclude an effective chemotherapy when there are: 1) vacuolar changes of carcinoma cells, 2) bizarre or multiple nuclei of carcinoma cells, 3) granulation tissue containing foam cells and/or 4) diffuse fibrosis. In the present study, the degree of effectiveness was evaluated conforming to the scheme defined by the Rules, i.e., dividing into Grades $0,1 \mathrm{a}, 1 \mathrm{~b}, 2$ and 3 according to how much and to what extent carcinoma has undergone such changes (Table 1). Since, in this grading, Grades 1 and 2 were defined as differing not qualitatively but in the area of cancer involved in the above changes, we performed morphometric evaluation in one case of Grade 2 using a digital image analyzer (model 9816; Hewlett Packard, Fort Collins, CO, USA) : the ratio of degenerative to the total area of cancer was determined by measuring respective areas in microscopic slides.

Two significance tests were applied to the result of evaluation: Wilcoxon's rank sum test to compare the difference in the grades between the IHC and non-IHC groups, and Spearman's rank correlation test, to relate the clinically assessed effects with the histological grading.

\section{Results}

Exhibited in Table 2 are the microscopic findings of the resected stomachs. The histological type of carcinoma was, according to the classification defined in the above Rules, tubular adenocarcinoma well differentiated in one case, moderately differentiated in seven, poorly differentiated adenocarcinoma in four and mucinous adenocarcinoma in one. Between the IHC and non-IHC groups, there was no marked unevenness with regard to the histological types. The extent of tumor proved to be up to the serosal surface in three cases, the subserosa in eight and the proper muscular layer in two. Regional lymph nodes were involved in eight cases. Based on these, a postsurgical staging of Stage II was given to five cases, and Stage III to the remaining eight.

The most remarkable finding in this table is that in the IHC group, there are three patients in whom we gave an evaluation of either Grade 3 (complete disappearance of tumor) or Grade 2 (remarkable degeneration), while in the 


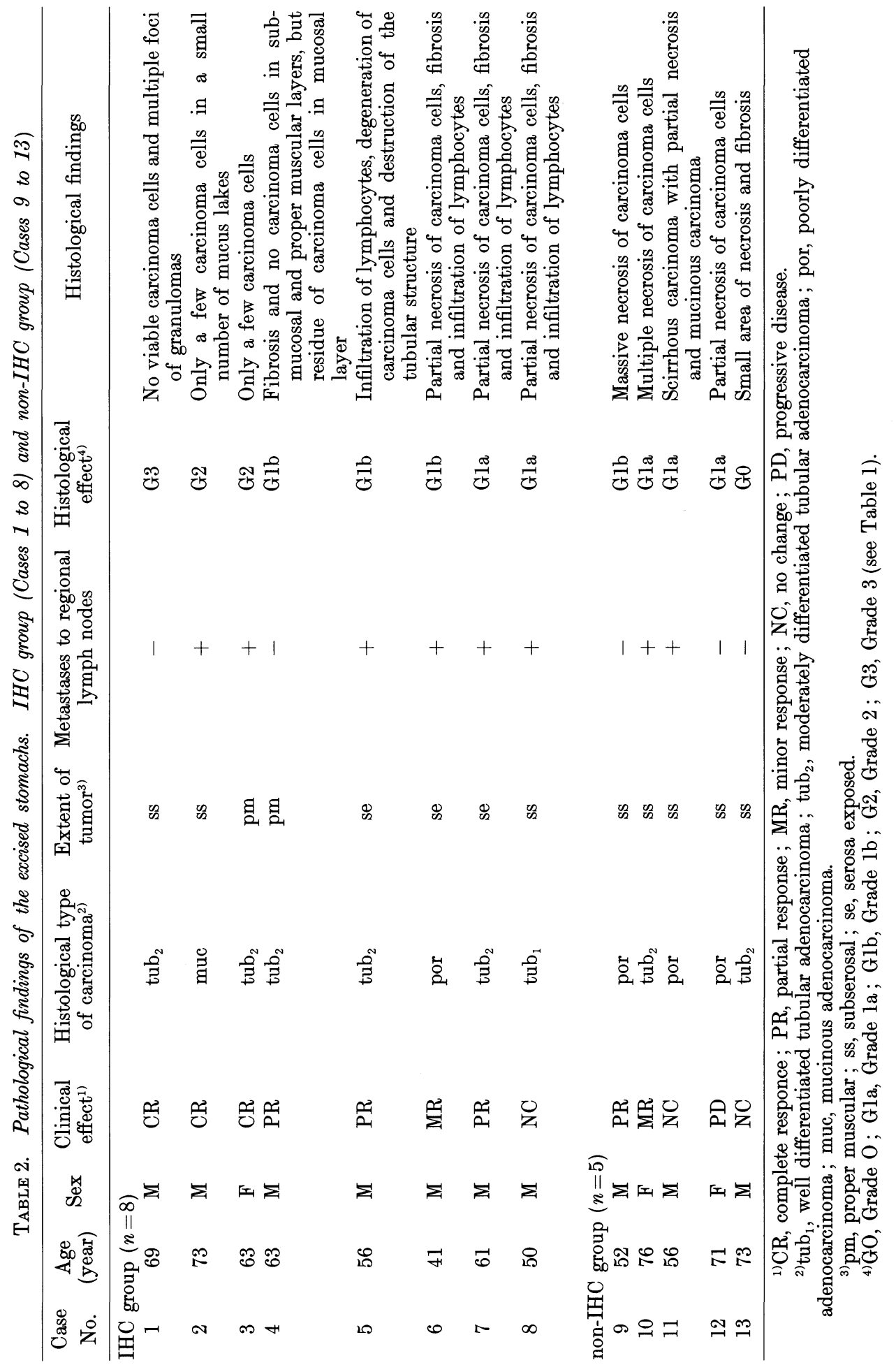


non-IHC group there was not a single patient given these ratings. In the following we demonstrate the microscopic finding in the three patients who showed such prominent effects.

Case 1. Male, aged 69. At an endoscopy performed for the patient's complaint of dysphagia, a crater-forming carcinoma of Borrmann's III type was detected directly below the cardia. Presurgical mucosal biopsy disclosed a moderately differentiated adenocarcinoma invading over the cardia into the lowest portion of esophagus (Fig. 1a). The patient received IHC according to the protocol, and then was subjected to a total gastrectomy 76 days after chemotherapy was begun. The resected stomach, macroscopically revealing little more than a shallow ulceration just below the cardia, proved microscopically to have a dense scar under the ulceration, which was extending across the whole layer of the gastric wall and into the esophageal submucosa (Fig. 1b). There was no evidently viable carcinoma cell. The scar contained over its entire area multiple foci of granulomas with lymphoid cells and swollen histiocytes (Fig. 1c), which were

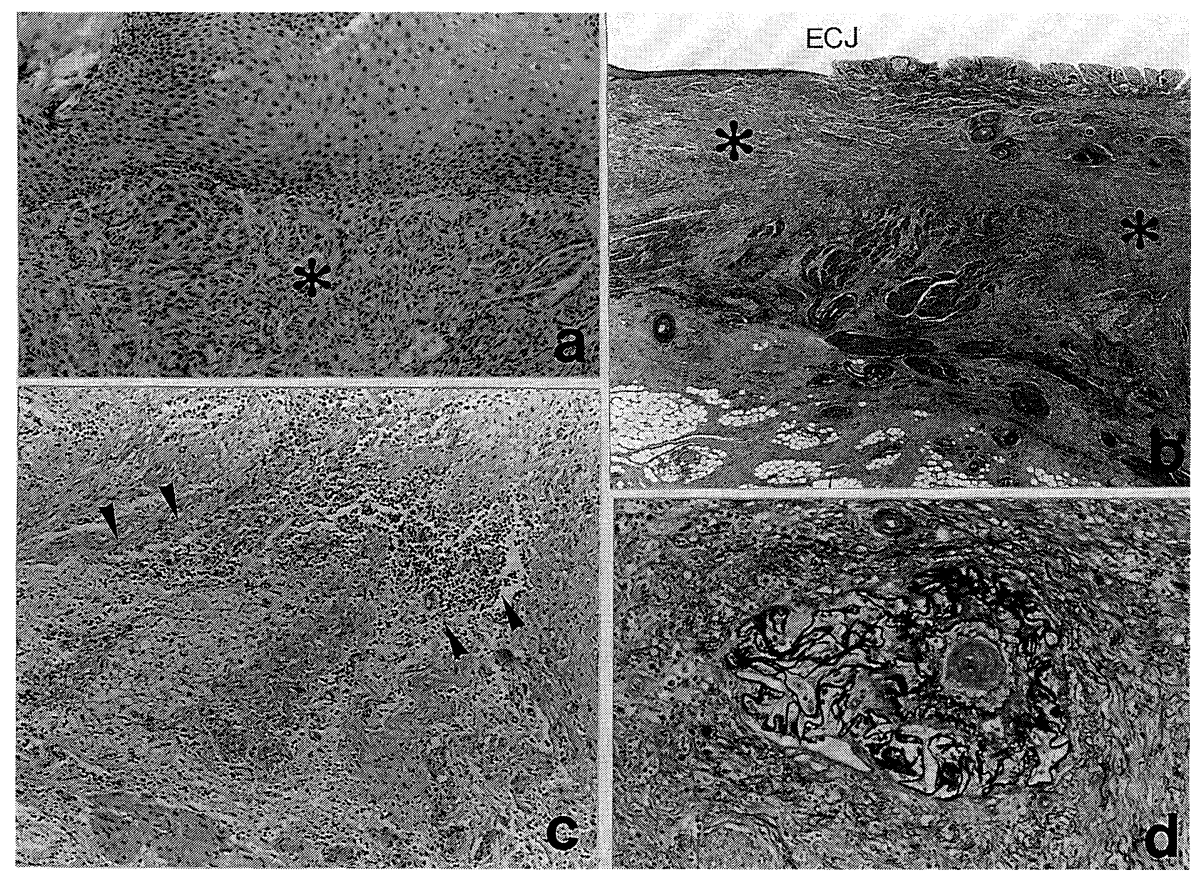

Fig. 1. Gastric histopathology in Case 1. a: Biopsy specimen taken before the introduction of IHC, with adenocarcinoma invading into the esophageal wall $(*) . \times 70$. b: The wall of the excised stomach involved in broad fibrosis (*) leaving the muscularis propria. $\times 8 . \quad \mathrm{c}:$ Granulomatous foci comprizing swollen macrophages (arrowheads) leaving no viable carcinoma. $\times 70$. d: "Crumpled" host arteries in the fibrotic area once infiltrated by carcinoma. $\times$ 70. 
interpreted as the remain of tissue reactions against carcinoma. Thus, the extensive scar was regarded as exactly corresponding to the area once invaded by carcinoma which, by the time of surgery, had been destroyed without leaving viable cells. In the fibrous area, there were small arteries showing aneurysm-like ectasia and crumpling as in Fig. 1d, which we think, as will be mentioned, are quite specific of the host arteries left in carcinoma infiltrating in their surroundings. The luminal ectasia is attributable to the loss of medial smooth muscles due to an impeded plasmal flow across the wall. That the subserosal vessels are involved in these changes strongly suggests the extention of carcinoma once involving as far as the subserosa. These considerations justified to give an assessment of Grade 3.

Case 2. Male, aged 73. A crater-forming carcinoma of Borrmann's III type was found in this patient. The tumor was situated in the upper corpus and along the lesser curve. Presurgical mucosal biopsy revealed a typical mucinous adenocarcinoma with mucus lakes containing dense aggregates of signet ring cells (Fig. 2a). With the introduction of IHC, the crater began to shrink and on the 93rd day, the state was clinically assessed as Complete Response (CR), when all that remained of the tumor was endoscopically only a small scar. A total gastrectomy was performed on the 163rd day. Although histologically mucus lakes were found remaining in the whole depth of the gastric wall, most of these were empty, leaving only a few carcinoma cells in a small number of lakes (Fig. 2b). Acellular lakes were found in lymph nodes too. The emptying, being likely to suggest the effect of drugs accumulated in the lakes, urged us to give a rating of Grade 2.
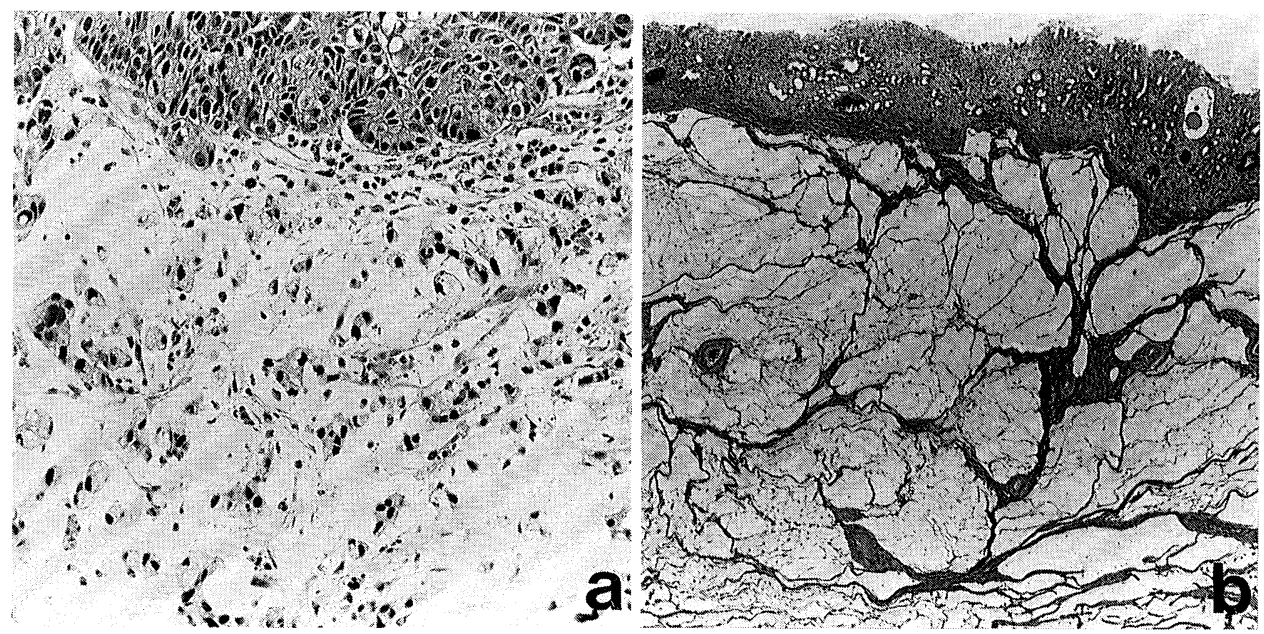

Fig. 2. Gastric histopathology in Case 2. a : Pre-chemotherapy biopsy, showing abundant carcinoma cells in mucus lakes. $\mathrm{b}$ : Surgical material with mucus lakes mostly cell-free. 
Case 3. Female, aged 63. With a complaint of epigastralgia, weight loss and anemia, she was found to have a carcinoma of Borrmann's III type below the cardia. IHC was introduced and on the 18th day, the tumor proved to have undergone a remarkable shrinkage. On biopsy which disclosed no carcinoma cells but only granulomatous changes, the patient was clinically assessed as to be in a CR state. A total gastrectomy was performed 64 days after chemotherapy started. Macroscopically, the resected stomach showed only a small ulceration in the subcardiac posterior wall. Microscopic examination of this portion exhibited a scar extending over the submucosal and muscular layers, at the center of which there was a small focus of adenocarcinoma, moderately differentiated, $5.8 \times 4.2 \mathrm{~mm}$ in area. Otherwise, there were no viable carcinoma cells. The scar contained several granulomas with histiocytes having swollen, vacuolated cytoplasm, that closely resembled those in Case 1 and were considered to be a host reaction against carcinoma cells (Fig. 3a). Degenerative signs like vacuolization were also found in carcinoma cells themselves, around which, neutrophils and lymphocytes were
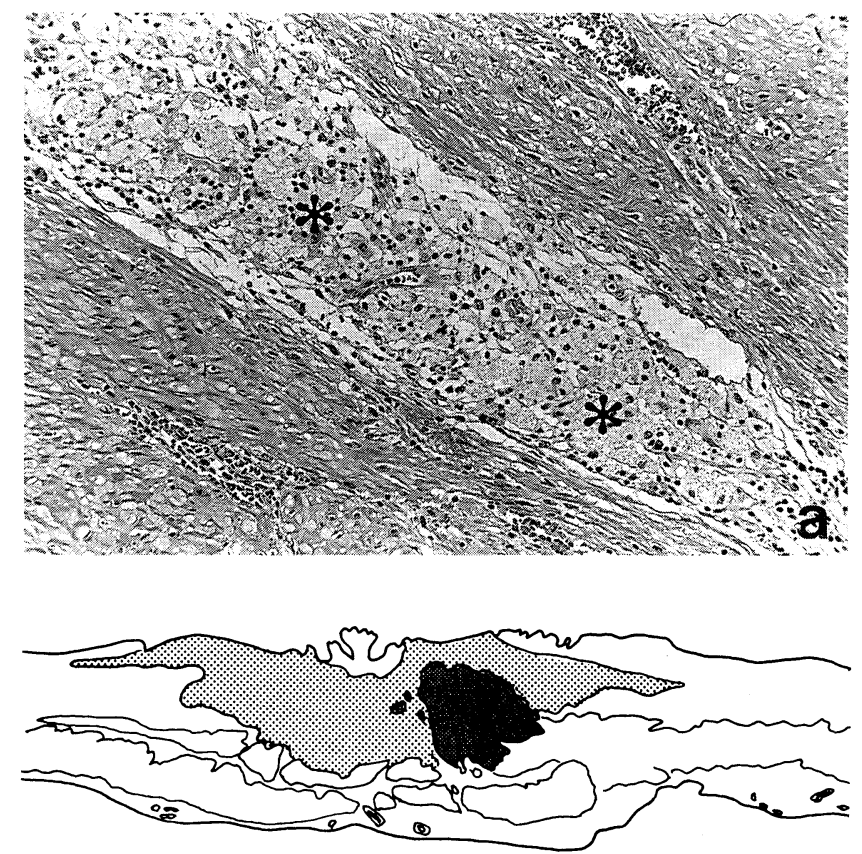

Carcinoma cells

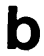

Fig. 3. Surgically excised stomach in Case 3. a: Granulomatous area in tunica muscularis, with hydropic macrophages. $\mathrm{b}$ : Tracing of gastric wall in a section with the maximum spread of carcinoma. Compare with the extension of fibrosed area. 


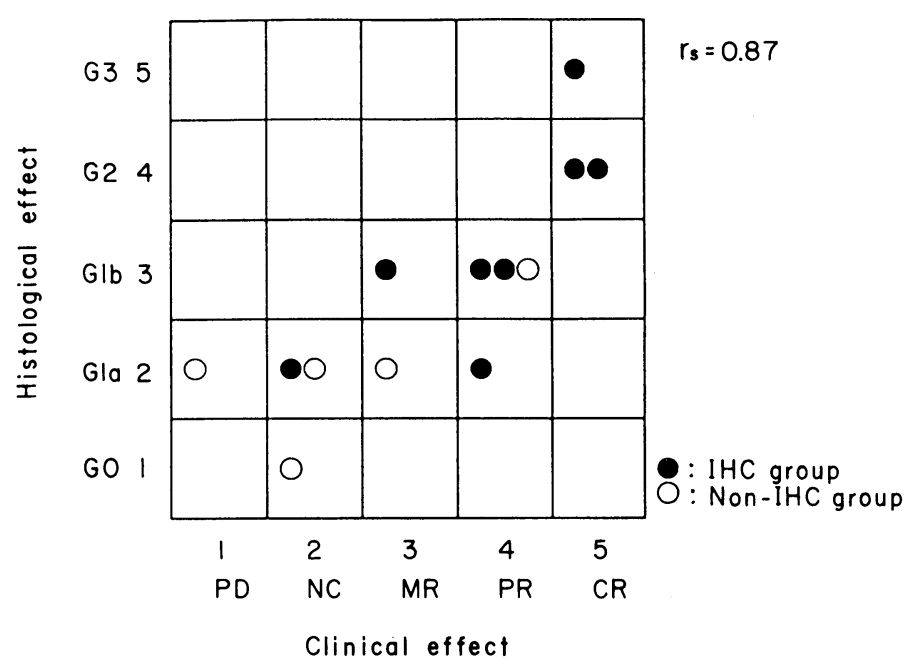

Fig. 4. Correlation between the clinical (abscissa) and the pathological (ordinate) assessments on the effect of chemotherapy, both expressed in a semiquantitative scaling. For explanations see text.

infiltrating. These were all regarded as histological expression of effective cancer chemotherapy. In the scar, there were also ectatic arteries with crumpling, suggesting that it corresponds to the original extent of carcinoma, the larger part of which was destroyed by chemotherapy. In the sections containing carcinoma, the area of viable tumor was measured and was compared with that of the total fibrotic area including tumor (Fig. 3b). The areal ratio of the viable tumor to the total was calculated at 0.21 , justifying to give a histological evaluation of Grade 2 , since the Rule requires for this rating a destruction of carcinoma in more than $2 / 3$ sectional area.

Prior to making statistical analysis, the result of histological assessment was expressed in a scatter diagram of Fig. 4 . Here the abscissa is scaled with the clinical effect of chemotherapy evaluated in five steps from Progressive Disease (PD) to Complete Response (CR), adding Moderate Response (MR) between No Change (NC) and Partial Response (PR). The ordinate expresses the histological grading, also in five grades. It appears that the patients subjected to IHC tend to have a higher histological rating. Also, as a whole, there appears to be a rough correlation between the clinical and the histological ratings.

The difference between the IHC and the non-IHC groups in the grade of histological effectiveness was subjected to Wilcoxon's rank-sum test. It turned out that the mean of the grade values is significantly higher in the IHC than in the non-IHC group $(p<0.05)$. The relation between the histological and clinical effects was examined by Spearman's rank-correlation test. The correlation coefficient $\left(r_{\mathrm{s}}\right)$ was calculated at 0.87 , which also proved to be significant $(p<0.1)$. 


\section{Discussion}

So far, many studies have been performed from various viewpoint on the chemotherapy of gastric carcinoma. However, discrepancies remain so much over its effect that as yet, we have no clear conclusion on its clinical significance. On one hand, some argues that for this disease, there is no effective therapy other than a surgical resection of the stomach (Adam and Efron 1989). On the other, there is a great difference among the reports on the effect of chemotherapy, even when performed on a same regimen (Cullinan et al. 1985; Preusser et al. 1989). Such ambiguities and inconsistencies may be explainable from the fact that as yet, a basis has not been established on which to evaluate the effect of chemotherapy in an objective and reproducible way. Particularly, the standard for histopathological assessment awaits further supplementation.

In microscopically evaluating the effect of chemotherapy, one faces difficulty mainly in two facets. The first barrier lies in the fact that there is little specificity in the chemotherapy-related changes of cancer. Anticancer drugs may cause degeneration and necrosis of carcinoma cells and leave fibrosis in the areas where carcinoma was invading, but these can also develop spontaneously due to ischemia to which cancer tissue is especially vulnerable. Simple coagulation necrosis of carcinoma cells not accompanied by inflammatory or granulomatous reaction was regarded as suggestive of ischemic changes. But one often encounters cases in which this criterion does not give a clear distinction. In determining whether a regimen of chemotherapy is really effective, we should be able to rely on information from more various sources than we had so far. For instance, one may expect some specific pattern in the distribution of chemotherapy-mediated necrosis which in some way differs from ischemic necrosis of carcinoma.

The other difficulty is related with the method of assessment. In the previous studies dealing with the effect of chemotherapy in patients with gastric carcinoma, evaluation has usually been based only on a gross clinical assessment of tumor volume and not on histopathological examination. Moreover, it is only in a very small number of reports that microscopic findings were taken into consideration. There has not been a single report in which the result of evaluation was subjected to a statistical treatment, that, in the present study, we did on as many as thirteen cases. Without comparing among a certain number of cases and bringing the result to statistical tests, confusion on the effect of chemotherapy cannot be overcome. In this sense, our study presents an unprecedented trial. Though the method we used is a semi-quantitative assessment, a significant difference proved to exist between the IHC and non-IHC groups. We also performed morphometry, though only in one patient. If introduced on a larger scale, it may help draw a more reliable conclusion.

Especially worth attention is the presence in the IHC group of a patient who earned not only a clinical assessment of CR but also a histological rating of Grade 
3 (Case 1). On a preoperative biopsy, the patient was demonstrated to have an adenocarcinoma infiltrating continuously into the lower esophagus. In the resected stomach, there were signs that cancer had once extended over the whole thickness of the gastric wall, leaving however no viable carcinoma cells but for an extensive fibrosis. One may say that the tumor was in reality an early mucosal carcinoma which was ulcerated, leaving such an extensive scar. However, we exclude this possibility based on a peculiar finding of small arteries running in the fibrous area : an aneurysm-like ectasia and crumpling, which, as demonstrated by Yaegashi and Takahashi (1990), are changes specific of host arteries remaining in carcinoma infiltration. Even in such cases, it may be difficult to draw a conclusion of complete cure by chemotherapy alone, because there is always room for a small number of carcinoma cells to escape detection by routine histopathology. Still, this case presents a remarkably successful chemotherapy which managed to bring a state of no longer confirmable carcinoma cells. Such cases are found only in a very small number of reports (Mizumoto et al. 1989; Tsujinaka et al. 1989; Uesugi et al. 1989; Yasutake et al. 1990). Furthermore, we have another two patients in the IHC group in whom the effect of chemotherapy was assessed as being Grade 2, a rating which was not given to any patient in the non-IHC group. Our results, combined with the statistical tests, are likely to be showing an increased effect of chemotherapy performed under angiotensin-induced hypertension.

\section{References}

1) Adam, Y.G. \& Efron, G. (1989) Trends and controversies in the management of carcinoma of the stomach. Surg. Gynecol. Obstet., 169, 371-385.

2) Cullinan, S.A., Moertel, C.G., Fleming, T.R., Rubin, J.R., Krook, J.E., Everson, L.K., Windschitl, H.E., Twito, D.I., Marschke, R.F. \& Foley, J.F. (1985) A comparison of three chemotherapeutic regimens in the treatment of advanced pancreatic and gastric carcinoma, fluorouracil vs. fluorouracil and doxorubicin vs. fluorouracil, doxorubicin, and mitomycin. J.A.M.A., 253, 2061-2067.

3) Japanese Research Society for Gastric Cancer (1985) The General Rules for the Gastric Cancer Study. 11th ed., Kanehara, Tokyo. (in Japanese)

4) Mizumoto, M., Ooue, M., Takami, Y., Iihara, K., Kurokawa, E., Akashi, H., Aoki, Y., Mori, H. \& Okano, K. (1989) An autopsied case of an aged patient with an advanced gastric cancer that showed a complete response to immunochemotherapy. Clin. Oncol., 35, 1791-1794. (in Japanese with English summary)

5) Preusser, P., Wilke, H., Achterrath, W., Fink, U., Lenaz, L., Heinicke, A., Meyer, J., Meyer, H.-J. \& Buente, H. (1989) Phase II study with the combination etoposide, doxorubicin, and cisplatin in advanced measurable gastric cancer. J. Clin. Oncol., 7, 1310-1317.

6) Sato, H., Wakui, A., Hoshi, M., Kurihara, M., Yokoyama, M. \& Shimizu, H. (1991) Randomized controlled trial of induced hypertension chemotherapy (IHC) using angiotensin II human (TY-10721) in advanced gastric carcinoma (TY-10721 IHC Study Group Report). Jpn. J. Cancer Chemother., 18, 451-460. (in Japanese)

7) Suzuki, M., Hori, K., Abe, I., Saito, S. \& Sato, H. (1981) A new apoproach to cancer chemotherapy: Selective enhancement of tumor blood flow with angiotensin II. $J$. 
Natl. Cancer Inst., 67, 663-669.

8) Suzuki, M., Hori, K., Abe, I., Saito, S. \& Sato, H. (1984) Functional characterization of the microcirculation in tumors. Cancer Metastasis Rev., 3, 115-126.

9) Suzuki, M., Takahashi, T. \& Sato, T. (1987) Medial regression of tumor-bearing host arteries and its functional significance. A morphometric study of hepatic arteries in human livers with hepatocellular carcinoma. Cancer, 59, 444-450.

10) Tsujinaka, T., Murata, A., Ogawa, M., Kido, Y., Shiozaki, H., Miyamoto, T., Kanbayashi, J., Kobayashi, K., Tahara, H., Miyata, M. \& Mori, T. (1989) The effect of EAP (Etoposide, Adriamycin, Cis-Platinum) on patients with advanced gastric cancer : The role of EAP as a neoadjuvant chemotherapy and histopathological study on its effect. Cancer Chemother., 16, 3295-3298. (in Japanese with English summary)

11) Uesugi, H., Azuumi, Y., Seigenji, K., Okabe, H., Mieno, H. \& Motoori, T. (1989) A case of advanced gastric cancer (Borrmann's Type 2) that disappeared following short term preoperative chemotherapy with UFT. Clin. Oncol., 35, 963-969. (in Japanese with English summary)

12) Yaegashi, H. \& Takahashi, T. (1990) Encasement and other deformations of tumorembedded host arteries due to loss of medial smooth muscles. Cancer, 65, 1097-1103.

13) Yasutake, K., Imamura, Y., Yoshimura, Y., Oya, M., Matsushita, K., Tokisue, M., Ohno, S. \& Masuda, T. (1990) A study of combined chemotherapy with MMC, ADM, CDDP, Etoposide (VP-16), 5'DFUR (MAC-VC therapy) in advanced cancer and local relapse of the stomach. Cancer Chemother., 17, 397-401. (in Japanese with English summary) 